\title{
The far-ultraviolet emission of early-type galaxies
}

\author{
J.-M. Deharveng, A. Boselli, and J. Donas \\ Laboratoire d'Astrophysique de Marseille, Traverse du Siphon, Les Trois Lucs, BP 8, 13376 Marseille Cedex 12, France \\ Received 20 March 2002 / Accepted 12 July 2002

\begin{abstract}
We have assembled a $U V$-flux selected sample of 82 early-type galaxies and collected additional information at other wavelengths. These data confirm a large spread of the $U V-V$ color in the range 2 to 5 . The spread in $U V-V$ is accompanied by a spread in $B-V$ that is mainly attributed to the range of morphological types and luminosities. A large fraction of the objects have red colors, $U V-V=4 \pm 0.4$, corresponding to a weak $U V$-upturn as observed with IUE. If the current interpretation for the $U V$ emission from early-type galaxies is applicable to our sample, the PAGB (Post-Asymptotic Giant Branch) tracks are the most common evolution path for the low-mass stars responsible for the $U V$ emission. A small number of very blue $(U V-V<1.4)$ objects have been found that can be reasonably interpreted as harbouring some low level of star formation. In contrast to a previous sample based on IUE observations, no correlation is found between the $U V-V$ color and the $\mathrm{Mg}_{2}$ spectral line index; possible explanations are reviewed. The potential of a more extended $U V$ survey like GALEX is briefly presented.
\end{abstract}

Key words. stars: AGB and post-AGB - stars: horizontal-branch - galaxies: stellar content - galaxies: elliptical and lenticular, $\mathrm{cD}$ - ultraviolet: galaxies

\section{Introduction}

The $U V$ emission discovered in early-type galaxies as early as 1969 by the Orbiting Astronomical Observatory-2 (Code et al. 1972) is now currently interpreted in terms of low-mass, helium-burning stars in extreme horizontal branch and subsequent stages of evolution. O'Connell (1999) has extensively reviewed the built-up of that interpretation thanks to the combination of high quality $U V$ data and new generations of theoretical models for advanced stellar evolution (e.g. Greggio \& Renzini 1990, 1999 and references therein). The former include spectra with the Hopkins Ultraviolet Telescope (HUT) (e.g. Ferguson \& Davidsen 1993; Brown et al. 1995) and high angular resolution images with HST (e.g. Brown et al. 1998, 2000). Notorious difficulties for converging on a well accepted interpretation were the large variety of advanced stages of stellar evolution and the sensitivity of $U V$ production to small changes of physical properties.

Because the most detailed observations are both time consuming and intrinsically difficult, the current interpretation of the far- $U V$ radiation from early-type galaxies relies on a small number of objects. In addition to the bulge of M 31, only 7 elliptical galaxies were spectroscopically observed with HUT; it is not yet possible to resolve $U V$-bright stars down to the horizontal branch beyond M 31 (bulge) and its companions (Brown et al. 2000). Studying a large sample of early-type galaxies would therefore require the use of cruder approaches,

Send offprint requests to: J.-M. Deharveng,

e-mail: jean-michel.deharveng@astrsp-mrs.fr such as broad-band and integrated $U V$ fluxes, but would still be of interest. It would help to understand the generality of the conclusions reached and to distinguish the possibility and frequency of low level of star formation in the population of early-type galaxies. Although this latter phenomenon is now excluded as a general explanation for the $U V$ emission, it may well be present in a number of objects and have implications on galaxy evolution.

A sample of 32 early-type galaxies was already studied by Burstein et al. (1988) (hereafter BBBFL) and, albeit observed spectroscopically with IUE, was mostly discussed in terms of their $(1550-V)$ color (see also Dorman et al. 1995). With the availability of several $U V$ imaging surveys performed in the IUE-era (Brosch 1999; O'Connell 1999), it is now possible to study a larger sample of early-type galaxies in the far- $U V$. Such a sample would have the advantage to be essentially $U V$ flux selected and to potentially reveal $U V$ emission from unexpected early-type objects. This is a significant difference with the BBBFL sample made of objects with substantial record in the refereed literature and selected for one-by-one spectroscopic investigation. An additional motivation of our approach is to prepare ourselves to the extended $U V$ survey of GALEX (Martin et al. 1999) and what should be learnt of the early-type galaxies.

The paper is organized as follows. Section 2 describes how our sample of $U V$ selected early-type galaxies has been built and is complemented by a wealth of data at other wavelengths. The $U V-V$ color distribution and color-color diagram are presented in Sects. 3 and 4. The analysis follows in Sect. 5. 
We first take advantage of the fact that the BBBFL sample contains most of the objects that have been studied in details to emphasize a possible relationship between the $U V$ color and the categories of stars responsible for the $U V$ radiation. We then discuss the relative frequencies of these categories of stars in the population of early-type galaxies, the possible cases of recent star formation, the role of global properties such as the luminosity, the relation with the $\mathrm{Mg}_{2}$ spectral line index and the $U V$ light profiles in a few objects.

\section{The sample}

\subsection{Data origin}

The sample analysed in this work is composed of all the optically selected early-type galaxies (type $\leq$ S0a) belonging to the Zwicky catalogue (CGCG, Zwicky et al. 1961-1968) $\left(m_{\mathrm{pg}} \leq 15.7\right)$ detected in the $U V$ by the FOCA experiment during the observations of the Coma, A1367 and Cancer clusters (Donas et al. 1990, 1995, private communication). To these, we add all the early-type galaxies belonging to the Virgo Cluster Catalogue (VCC; Binggeli et al. 1985) $\left(m_{\mathrm{pg}} \leq 18\right)$ detected by SCAP, FOCA and FAUST in the direction of Virgo (Donas et al. 1987, private communication; Deharveng et al. 1994). The sample is thus composed primarily of cluster galaxies, even though some background or foreground objects are also included. Galaxies whose $U V$ detection is doubtful because of confusion with nearby objects, (such as VCC 311), unless specified, have been systematically excluded. The sample, largely dominated by objects observed with FOCA (85\%), is complete to a $U V$ magnitude of about 18 whereas only 7 objects come from the less deep images of SCAP and FAUST. Two additional early-type galaxies identified by Brosch et al. (1997) in their detailed study of FAUST images in the direction of the Virgo cluster have not been included. In order to preserve homogeneity and $U V$-flux selection, the sample was not extended with other sources of $U V$ data (UIT archives, O'Connell et al. 1992; Maoz et al. 1996; Rifatto et al. 1995 and references therein).

The final combined sample comprises 82 early-type galaxies, including a few dwarf ellipticals and spheroidals. The accuracy of the morphological classification is excellent for the Virgo galaxies (Binggeli et al. 1985). Because of the higher distance, the morphology of galaxies belonging to the other surveyed regions suffers from an uncertainty of about 1.5 Hubble type bins.

\subsection{UV data and precision}

Most of the $U V$ data are total integrated magnitudes obtained at $2000 \AA$ with the FOCA experiment described by Milliard et al. (1991). The FOCA $U V$ magnitudes from Donas et al. (1990, 1995) have been reprocessed adopting a new zero-point calibration and a revised version of the data reduction pipeline (Donas et al., private communication). A comparison of the FOCA magnitudes with IUE data (stars and galaxies) has revealed large fluctuations from object to object, with the FOCA fluxes being on average 0.3 mag brighter. Because of this dispersion and various possible explanations on a case by case basis, we decided to stay on the FOCA calibration in order to be consistent with previous works. The comparison with IUE will again be addressed in the specific context of the colors of the galaxies in Sect. 3. The $U V$ magnitudes at $1650 \AA$ of the additional galaxies (6) from FAUST have been transformed to $2000 \AA$ using the relation $U V(2000 \AA)=U V(1650 \AA)+$ 0.2 . This relation is intended to account for the average spectral trend of ellipticals between $1650 \AA$ and $2000 \AA$ as well as the comparison of FAUST magnitudes with other $U V$ measurements (Deharveng et al. 1994). The estimated error on the (FOCA) $U V$ magnitude due to the flux extraction procedure and to the linearisation of the photographic plates is $0.3 \mathrm{mag}$ in general, but it ranges from $0.2 \mathrm{mag}$ for bright galaxies to 0.5 mag for weak sources. This, combined with the previously discussed uncertainty on the zero point, gives errors on the $U V$ magnitudes of $\sim 0.5 \mathrm{mag}$. This uncertainty should be reminded when discussing color trends in our sample; it is extremely large in comparison with the current range of optical colors (as $B-V$ ) but should be seen in the context of the much larger range of variation of the $U V$ color. A comparison of 4 galaxies measured with both FOCA and FAUST (for homogeneity only the FOCA data have been retained in the sample) shows the FOCA fluxes 0.55 mag fainter than FAUST fluxes on average. This number suggests a possible systematic effect but remains consistent with our evaluation of the uncertainty of $U V$ magnitudes.

\subsection{Complementary data}

Optical data, available for 63 objects in the $V, 72$ in the $B$ and 51 in the $U$ band are from Gavazzi \& Boselli (1996) and Boselli et al. (private communication). NIR data, from Nicmos3 observations, are taken from Boselli et al. (1997) and Gavazzi et al. (2000a, 2001) (74 galaxies). From these data we derive total (extrapolated to infinity) magnitudes $H_{\mathrm{T}}$ determined as described in Gavazzi et al. (2000b) with typical uncertainties of $\sim 10 \%$. For a few objects we derive the $H$ luminosity from $K$ band measurements assuming an average $H-K$ color of $0.25 \mathrm{mag}$ (independent of type; see Gavazzi et al. 2000b) when the true $H-K$ color is not available. The estimated error on the optical and near-IR magnitudes is $0.1 \mathrm{mag}$.

The multifrequency data used in this work are listed in Table 1, arranged as follow:

- Column 1: VCC designation, from Binggeli et al. (1985) for Virgo galaxies, or CGCG (Zwicky et al. 1961-1968) for A1367, Coma and Cancer cluster galaxies.

- Column 2: UGC name.

- Column 3: NGC/IC name.

- Column 4: morphological type as given in the VCC for Virgo galaxies or in Gavazzi \& Boselli (1996) for the other objects.

- Column 5: photographic magnitude from the VCC for Virgo galaxies, from the CGCG for the other objects.

- Columns 6 and 7: major and minor optical diameters. For VCC galaxies the diameters are measured on the du Pont plates at the faintest detectable isophote. For CGCG galaxies these are the major and minor optical diameters $\left(a_{25}\right.$, 
$b_{25}$ ) (in arcmin) derived as explained in Gavazzi \& Boselli (1996).

- Column 8: distance, in Mpc. Distances to the various substructures of Virgo are as given in Gavazzi et al. (1999a). A distance of 91.3 and $96 \mathrm{Mpc}$ is assumed for galaxies in the A1367 and Coma clusters respectively. For Cancer cluster galaxies and for background and foreground objects the distance is determined from the redshift assuming $H_{0}=75 \mathrm{~km} \mathrm{~s}^{-1} \mathrm{Mpc}^{-1}$.

- Column 9: cluster membership as defined in Gavazzi et al. (1999a) for Virgo and in Gavazzi et al. (1999b) for A1367 and Coma. P is for pairs, $\mathrm{G}$ for groups, BkgV for galaxies in the background of Virgo, ForC for objects in the foreground of Coma.

- Columns 10 to 15: $K, H, J, V, B$ and $U$ magnitudes determined as in Gavazzi \& Boselli (1996), corrected for galactic extinction according to Burstein \& Heiles (1982). S0a galaxies are corrected for internal extinction as in Gavazzi \& Boselli (1996).

- Column 16 and 17: UV (2000 ̊) magnitude corrected for galactic extinction according to Burstein \& Heiles (1982) assuming $A(U V)=2.1 \times A(B)$ (all the targets being high galactic latitude objects, $A(U V) \leq 0.3 \mathrm{mag})$, and reference.

- Column 18: $\mathrm{Mg}_{2}$ data for the nuclear regions from Golev \& Prugniel (1998) and Jorgensen (1999). The index is defined as in Worthey (1994).

- Column 19: logarithm of the $H$ band luminosity, in solar units, determined from the relation $\log L_{H}=11.36+$ $2 \log D-0.4 H_{\mathrm{T}}$, where $H_{\mathrm{T}}$ is the total extrapolated $H$ magnitude and $D$ the distance (in $\mathrm{Mpc}$ ).

- Column 20: the $C_{31}$ index, defined as the ratio of the radii containing $75 \%$ to $25 \%$ of the total $\mathrm{H}$-band light of the galaxy.

- Column 21: comments to individual objects.

\section{The $U V-V$ color distribution}

The scatter of the early-type galaxies in the $U V$ in comparison with their behavior in the optical to near-infrared domain was noted as early as the first OAO-2 observations (Code \& Welch 1981). This scatter was confirmed by subsequent observations (e.g. Burstein et al. 1988), and it was shown as well that the scatter among $U V$ colors of galaxies with similar types was decreasing from early to late types (Code \& Welch 1982; Smith $\&$ Cornett 1982; Deharveng et al. 1994).

The distribution of the $U V-V$ colors of 63 galaxies of our sample are illustrated in Fig. 1 and compared with that of the $(1550-V)$ colors of BBBFL. The distributions are similar in several aspects: a peak near a color of 4 , a relatively sharp cut-off on the red side, a more shallow decrease on the blue side (from color 4 to 2) and a few "outliers" on the very blue side (precise definition and implications to be seen later on). In both cases the bulk of the objects have colors in the range 2 to 5 or so.

For five objects in common (NGC 4374, NGC 4406, NGC 4472, NGC 4486, NGC 4889), our $U V-V$ colors are found 1.4 mag redder on average than those of BBBFL. Three factors may account for this disturbingly large difference. First,
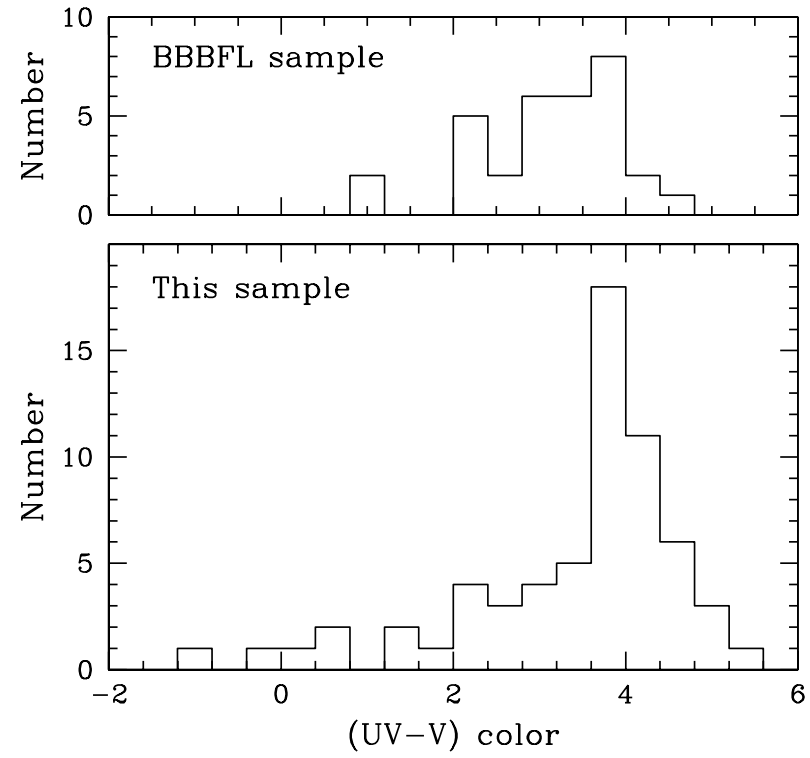

Fig. 1. Distribution of the $U V$ colors.

our $(2000-V)$ colors are less influenced than the $(1550-V)$ colors by the $U V$-upturn phenomenon. Second, our measurements are integrated whereas the IUE data are for a $10^{\prime \prime} \times 20^{\prime \prime}$ region centered on the nucleus, and all objects (except M 32) are known to become redder in $U V-B$ at large radii (Ohl et al. 1998). Last but not least, the $U V$ flux is integrated in a smaller area than the $V$ light in the case of objects that are well resolved with FOCA; for 3 of the five objects in common, this factor would reduce the $U V-V$ color by about $0.7 \mathrm{mag}$. For this reason and since the peak of our histogram (Fig. 1) appears only slightly redder than the BBBFL distribution, we remain confident that a large discrepancy in $U V-V$ colors is not the rule.

It is important to note that the increase in the number of objects from the blue to the peak at a color of $\sim 4$ is opposite to the trend expected from selection effects with $U V$-flux limited samples. These effects decrease the volume for finding red galaxies, hence their proportion relative to blue galaxies. This very trend certainly plays a role in the cutoff at color $>4$.

\section{Color-color diagram}

The scatter of the $U V-V$ color can be studied as a function of the $B-V$ color in the color-color diagram of Fig. 2. The objects can be roughly separated in two groups. A first group, forming a vertical plume with red $B-V$ colors $(>0.9)$, is consistent with the idea that the population of stars responsible for the $U V$ emission and whose changing proportions would explain the scatter in the $U V-V$ color are expected to make virtually undetectable contribution at visible wavelengths (O'Connell 1999). A second group is made of objects that get bluer in $B-V$ and $U V-V$. In addition to star formation, many factors such as the color-magnitude relation and the metallicity have already been identified as responsible for a scatter (or blueing) in the $B-V$ color of elliptical galaxies.

In this presentation we have ignored the fact that the $U V-$ $V$ and $B-V$ colors are not completely independent variables. 
Table 1. The sample galaxies: Virgo.

\begin{tabular}{|c|c|c|c|c|c|c|c|c|c|c|c|c|c|c|c|c|c|c|c|c|}
\hline $\begin{array}{r}\mathrm{VCC} \\
(1)\end{array}$ & $\begin{array}{l}\text { UGC } \\
(2)\end{array}$ & $\begin{array}{c}\text { NGC/IC } \\
\text { (3) }\end{array}$ & $\begin{array}{l}\text { type } \\
\text { (4) }\end{array}$ & $\begin{array}{l}m_{\mathrm{pg}} \\
(5)\end{array}$ & $\begin{array}{c}a \\
(6)\end{array}$ & $\begin{array}{l}b \\
(7)\end{array}$ & $\begin{array}{l}\text { Dist } \\
(8)\end{array}$ & $\begin{array}{c}\text { Cluster } \\
(9)\end{array}$ & $\begin{array}{c}K \text { mag } \\
(10)\end{array}$ & $\begin{array}{c}H \text { mag } \\
(11)\end{array}$ & $\begin{array}{c}J \text { mag } \\
(12)\end{array}$ & $\begin{array}{c}V \text { mag } \\
(13)\end{array}$ & $\begin{array}{c}B \text { mag } \\
(14)\end{array}$ & $\begin{array}{c}U \text { mag } \\
(15)\end{array}$ & $\begin{array}{c}U V \text { mag } \\
(16)\end{array}$ & $\begin{array}{l}\text { Ref. } \\
(17)\end{array}$ & $\begin{array}{l}\mathrm{Mg}_{2} \\
(18)\end{array}$ & $\begin{array}{c}\log L_{H} \\
(19)\end{array}$ & $\begin{array}{l}C_{31} \\
(20)\end{array}$ & $\begin{array}{l}\text { Note } \\
(21)\end{array}$ \\
\hline 49 & 7203 & 4168 & E & 12.21 & 1.76 & 1.40 & 32 & M & 8.32 & 8.84 & 9.62 & 11.72 & 12.63 & 13.11 & 12.50 & 2 & 0.246 & 10.89 & 3.71 & $*$ \\
\hline 288 & - & - & $\mathrm{dE}$ & 17.70 & 0.52 & 0.31 & 17 & $\mathrm{~N}$ & - & - & - & - & - & - & 16.82 & 8 & - & - & - & \\
\hline 355 & 7365 & 4262 & S0 & 12.41 & 1.87 & 1.63 & 17 & A & 8.37 & 8.59 & 9.33 & 11.62 & 12.56 & 13.10 & 14.93 & 8 & 0.294 & 10.38 & 5.72 & \\
\hline 389 & - & 781 & dS0 & 14.21 & 1.41 & 0.91 & 17 & A & - & - & - & - & - & - & 16.85 & 8 & - & - & - & \\
\hline 608 & - & 4322 & $\mathrm{dE}$ & 14.94 & 1.25 & 0.62 & 17 & A & 11.80 & 11.70 & 12.62 & 14.52 & 15.24 & 15.46 & 16.89 & 8 & - & 9.07 & 2.66 & \\
\hline 616 & - & 4325 & E & 14.40 & 1.55 & 0.97 & 102.8 & $\mathrm{BkgV}$ & - & - & - & 13.47 & 14.40 & 14.85 & 14.06 & 1 & - & - & - & \\
\hline 715 & - & 3274 & S0 & 14.80 & 0.93 & 0.46 & 90.9 & $\mathrm{BkgV}$ & - & - & - & - & - & - & 17.27 & 9 & - & - & - & \\
\hline 731 & 7488 & 4365 & E & 10.51 & 8.73 & 6.18 & 23 & B & 6.50 & 6.78 & 7.48 & 9.66 & 10.64 & 11.25 & 13.75 & 1 & 0.312 & 11.42 & 6.00 & $*$ \\
\hline 759 & 7493 & 4371 & S0 & 11.80 & 5.10 & 2.48 & 17 & A & 7.77 & 8.05 & 8.76 & 10.87 & 11.85 & 12.41 & 15.61 & 6 & - & 10.62 & 3.93 & \\
\hline 763 & 7494 & 4374 & $\mathrm{E}$ & 10.26 & 10.07 & 10.07 & 17 & A & 6.43 & 6.69 & 7.43 & 9.16 & 10.16 & 10.76 & 13.71 & 6 & 0.287 & 11.16 & 4.70 & $*$ \\
\hline 781 & 7500 & 3303 & dS0 & 14.72 & 1.08 & 0.50 & 17 & A & 12.11 & - & - & 14.34 & 15.03 & - & 16.92 & 6 & - & 8.94 & 3.04 & $*$ \\
\hline 828 & 7517 & 4387 & $\mathrm{E}$ & 12.84 & 1.84 & 0.83 & 17 & A & 9.04 & 9.32 & 10.03 & 12.29 & 13.19 & 13.74 & 16.67 & 6 & 0.228 & 10.08 & 4.25 & \\
\hline 870 & - & 3331 & $\mathrm{dS} 0$ & 15.52 & 1.16 & 0.43 & 185.7 & $\mathrm{BkgV}$ & - & - & - & - & - & - & 16.33 & 6 & - & - & - & \\
\hline 881 & 7532 & 4406 & E & 10.06 & 11.37 & 7.51 & 17 & A & 6.04 & 6.27 & 6.98 & 8.95 & 9.94 & 10.51 & 14.11 & 6 & 0.290 & 11.38 & 7.06 & $*$ \\
\hline 914 & - & - & $\mathrm{dE}$ & 19.00 & 0.25 & 0.25 & 23 & B & - & - & - & - & - & 20.02 & 17.29 & 9 & - & - & - & \\
\hline 944 & 7542 & 4417 & So & 12.08 & 3.60 & 1.00 & 23 & B & 8.21 & 8.42 & 9.20 & 11.13 & 12.03 & 12.52 & 15.58 & 9 & - & 10.89 & 12.52 & $*$ \\
\hline 951 & 7550 & 3358 & $\mathrm{dE} / \mathrm{dS} 0$ & 14.35 & 1.43 & 0.94 & 17 & B & - & 11.54 & - & 13.94 & 14.58 & - & 16.32 & 6 & - & 9.18 & 3.25 & \\
\hline 1003 & 7568 & 4429 & SOa & 11.15 & 8.12 & 3.52 & 17 & A & 6.54 & 6.74 & 7.43 & 9.59 & 10.58 & 11.22 & 14.98 & 6 & 0.232 & 11.01 & 5.48 & $*$ \\
\hline 1010 & 7569 & 4431 & dS0 & 13.68 & 1.58 & 0.79 & 17 & A & 10.53 & 10.74 & 11.35 & 13.23 & 14.05 & 14.59 & 16.91 & 6 & 0.153 & 9.57 & 2.86 & \\
\hline 1030 & 7575 & 4435 & S0 & 11.84 & 2.92 & 2.48 & 17 & A & 7.68 & 7.92 & 8.66 & 10.94 & 11.82 & 12.33 & 15.31 & 6 & 0.189 & 10.74 & 10.06 & $*$ \\
\hline 1111 & - & - & $\mathrm{dE}$ & 17.70 & 0.33 & 0.20 & 17 & A & - & - & - & - & - & - & 15.73 & 6 & - & - & - & \\
\hline 1125 & 7601 & 4452 & So & 13.30 & 2.92 & 0.57 & 17 & A & 9.04 & 9.31 & 10.10 & 11.91 & 12.87 & 13.41 & 16.23 & 6 & - & 10.06 & 2.67 & \\
\hline 1146 & 7610 & 4458 & E & 12.93 & 1.80 & 1.52 & 17 & A & 9.38 & 9.69 & 10.32 & 12.32 & 13.18 & 13.64 & 16.74 & 6 & 0.208 & 10.06 & 7.99 & \\
\hline 1226 & 7629 & 4472 & $\mathrm{E}$ & 9.31 & 10.25 & 8.11 & 17 & $S$ & 5.30 & 5.59 & 6.31 & 8.54 & 9.52 & 10.16 & 13.35 & 9 & 0.313 & 11.66 & 7.34 & $*$ \\
\hline 1250 & 7637 & 4476 & S0 & 12.91 & 1.89 & 0.94 & 17 & A & 9.50 & 9.82 & 10.47 & 12.41 & 13.23 & 13.55 & 15.33 & 6 & 0.141 & 9.88 & 3.59 & \\
\hline 1279 & 7645 & 4478 & $\mathrm{E}$ & 12.15 & 1.89 & 1.43 & 17 & A & 8.24 & 8.52 & 9.17 & 11.45 & 12.36 & 12.77 & 15.47 & 6 & 0.233 & 10.35 & 3.37 & \\
\hline 1297 & - & - & E & 14.33 & 0.51 & 0.45 & 17 & A & 10.07 & 10.34 & 11.13 & 13.44 & 14.42 & 15.03 & 17.41 & 6 & 0.290 & 9.72 & 3.52 & \\
\hline 1316 & 7654 & 4486 & E & 9.58 & 11.00 & 11.00 & 17 & A & 5.92 & 6.19 & 7.01 & 8.82 & 9.82 & 10.37 & 12.70 & 6 & 0.270 & 11.34 & 4.20 & $*$ \\
\hline 1327 & 7658 & - & E & 13.26 & 1.10 & 0.88 & 17 & A & 9.07 & 9.23 & 9.75 & 11.49 & 12.13 & 12.27 & 14.49 & 6 & - & 10.14 & 4.43 & $*$ \\
\hline 1368 & 7665 & 4497 & SOa & 13.12 & 2.01 & 0.85 & 17 & A & 9.60 & 9.72 & - & 12.18 & 13.05 & 13.46 & 17.22 & 6 & - & 9.85 & 2.81 & \\
\hline 1499 & - & 3492 & $\mathrm{E}$ & 14.94 & 0.64 & 0.46 & 17 & A & - & 12.59 & - & 14.77 & 15.26 & - & 13.79 & 1 & - & 8.79 & 2.74 & $*$ \\
\hline 1535 & 7718 & 4526 & So & 10.61 & 7.00 & 2.01 & 17 & $S$ & 6.37 & 6.65 & 7.47 & 9.83 & 10.80 & 11.36 & 14.04 & 1 & 0.272 & 11.19 & 10.59 & \\
\hline 1809 & 7825 & 3631 & SOa & 14.17 & 1.10 & 0.67 & 37.3 & $\mathrm{BkgV}$ & - & - & - & - & - & - & 10.70 & 1 & - & - & - & \\
\hline
\end{tabular}




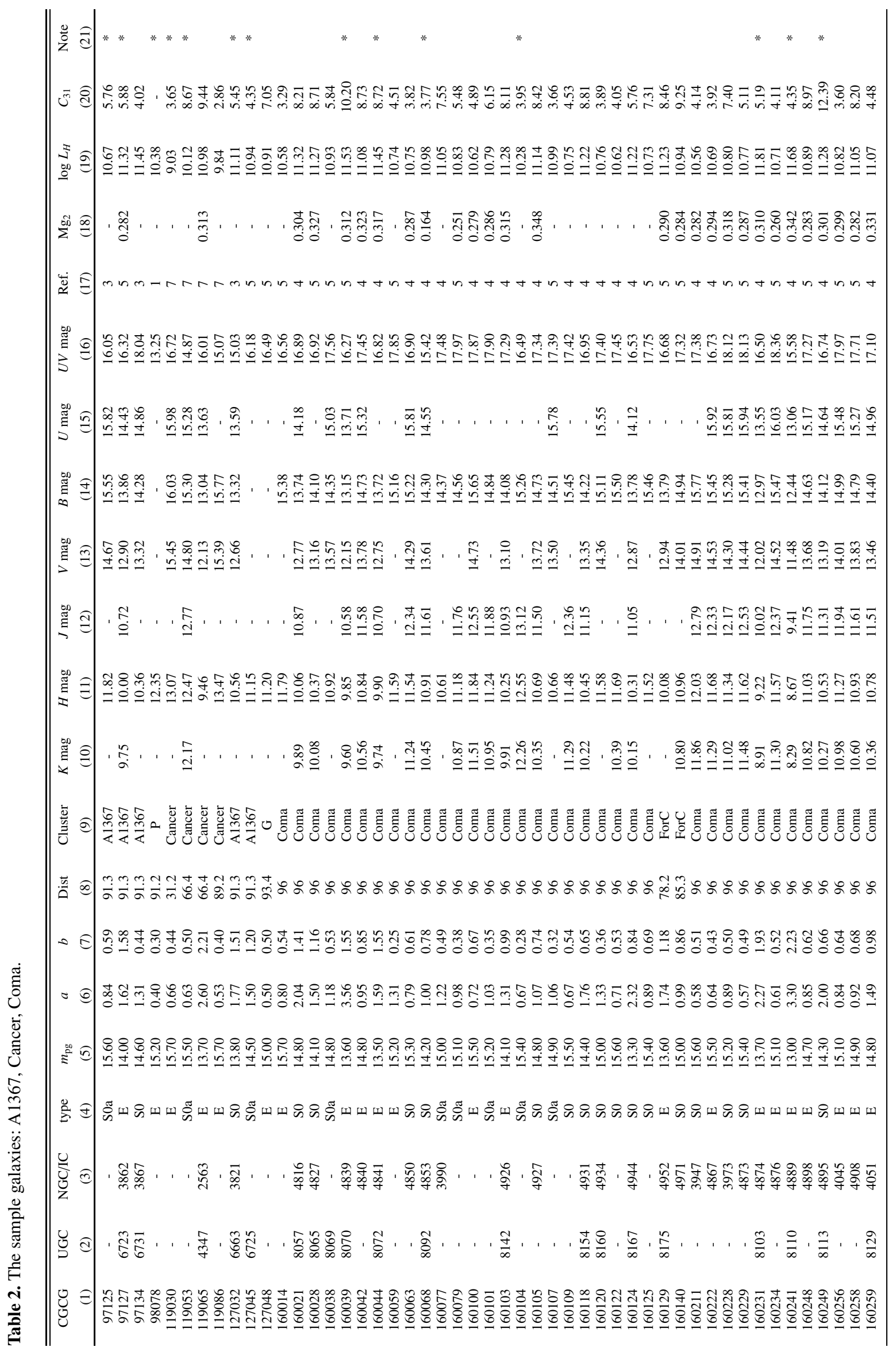


Comments to individual objects:

Virgo:

49: low-luminosity dwarf Seyfert nucleus (Ho et al. 1994).

731: $\mathrm{H} \alpha+[\mathrm{NII}] E W=2 \AA$ (Kennicutt \& Kent 1983).

763: Low Excitation Radio Galaxy (NED); H $\alpha+$ [NII]EW = 1 Å (Trinchieri \& Di Serego Alighieri 1991); also measured with FAUST (Deharveng et al. 1994).

781: interacting with NGC 4388? (Corbin et al. 1988).

881: M 86; $\mathrm{H} \alpha+[\mathrm{NII}] E W=13 \AA$ (Trinchieri \& Di Serego Alighieri 1991); also measured with FAUST (Deharveng et al. 1994).

944: classified "SB0: sp" in NED,

1003: $\mathrm{H} \alpha+[\mathrm{NII}] E W=5 \AA$ (Boselli \& Gavazzi 2002).

1030: interacting with NGC 4438; LINER.

1226: M 49; $\mathrm{H} \alpha+[\mathrm{NII}] E W=1 \AA$ (Kennicutt \& Kent 1983); also measured with FAUST (Deharveng et al. 1994).

1316: M 87; radio galaxy; H $\alpha+[\mathrm{NII}] E W=2 \AA$ (Boselli \& Gavazzi 2002); also measured with FAUST (Deharveng et al. 1994).

1327: bright star superposed (Prugniel et al. 1987).

1499: unresolved in $U V$ from the nearby companion VCC 1491; given the extreme red color of VCC 1491 and the blue color of VCC 1499 (Gavazzi et al. 2001 ), the $U V$ source

should be identified to VCC 1499 (instead of VCC 1491 in Deharveng et al. 1994). VCC 1499 is as blue as a dwarf irregular and has a spectrum with the characteristics of a post-starburst

galaxy (PSB) (Gavazzi et al. 2001).

Coma/A1367/Cancer:

97125: $\mathrm{H} \alpha+[\mathrm{NII}] E W=26 \AA$ (Moss et al. 1998), $\mathrm{H} \alpha+[\mathrm{NII}] E W=21 \AA$ (Gavazzi et al. 1998).

97127: Low Excitation Radio Galaxy (NED).

98078: Mrk 758, $\mathrm{H} \alpha+[\mathrm{NII}] E W=82 \AA$ (Gavazzi et al. 1998).

119030: classified "spiral" in NED.

119053: $\mathrm{H} \alpha+[\mathrm{NII}] E W=42 \AA$ (Kennicutt et al. 1984).

127032: classified as (R)SAB(s)ab in NED.

127045: $\mathrm{H} \alpha+[\mathrm{NII}] E W=13 \AA$ (Moss et al. 1998).

160039: radio galaxy (NED).

160044: binary system.

160068: AGN (NED); Balmer absorption lines (Sparke et al. 1980); PSB (Caldwell et al. 1993).

160104: PSB (Caldwell et al. 1997).

160231: cD galaxy.

160241: cD galaxy.

160249: classified as "SA0 pec sp" in NED.

References to the $U V$ data: 1: FAUST data (Deharveng et al. 1994); 2: SCAP data (Donas et al. 1987); 3 : Donas et al. (1990) (reprocessed data, $\Delta$ zero - point $=+0.30$ mag, see text);

4: Donas et al. (1995) (reprocessed data, $\Delta$ zero - point = -0.12 mag, see text); 5-9: Donas et al. private communication (5, Coma-A1367), (6, Virgo center), (7, Cancer), (8, Virgo M100),

(9, Virgo M 49). 


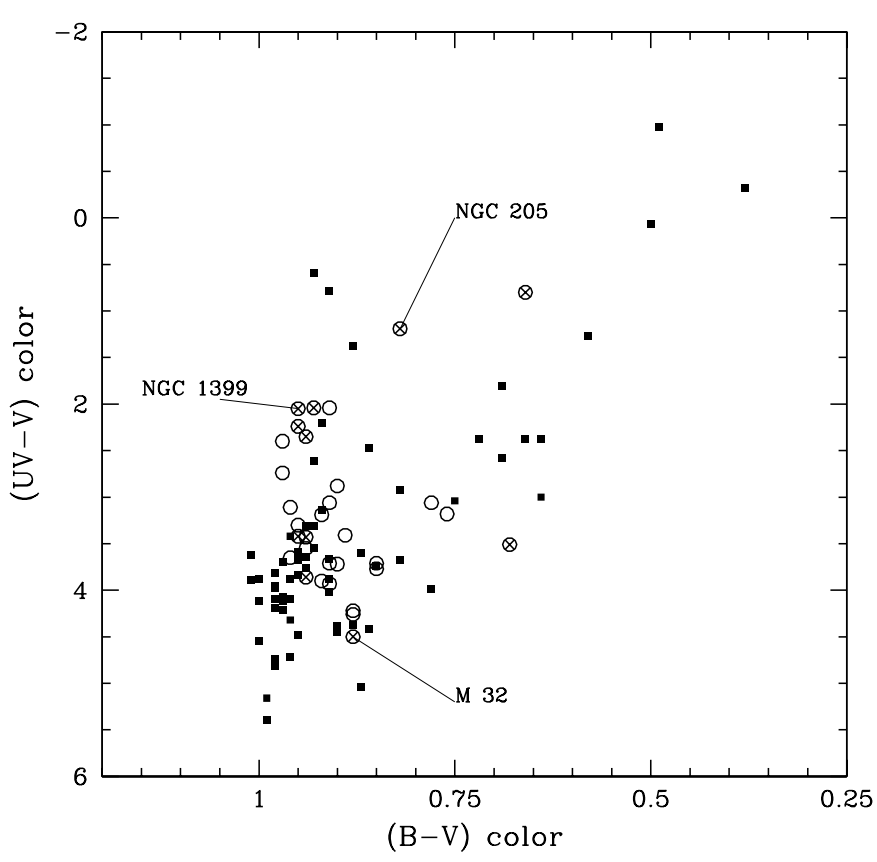

Fig. 2. $U V-V$ vs. $B-V$ color-color diagram for the galaxies of our sample (solid square) and the BBBFL sample (open circles). The 11 objects of the BBBFL sample which have been studied in details with HUT or HST and are used as references are marked as diagonal crosses. Great care should be taken when comparing the two samples because of differences between the two datasets (see text) and the fact that the $B-V$ refers to the integrated light. The specific issue of the $B-V$ of NGC 205 is detailed in Sect. 5.4.

We have verified that the results are similar in the $U V-B$ vs. $B-$ $V$ diagram but we keep using the $U V-V$ color for comparison with the previous work of BBBFL.

In spite of the differences in the observing wavelength and the measurement aperture, the BBBFL data have been plotted in Fig. 2 for comparison. These data do not populate the diagram in the same specific areas as our data (for instance a significant number of objects with $U V-V$ color as blue as 2 but red in $B-V$ are added) but follow the same trends. The $B-V$ colors needed to display the color-color diagram for the BBBFL galaxies have been obtained from the NASA Extragalactic Database. They refer to the integrated light whereas the $U V-V$ colors are for the IUE aperture, which may be a problem in some cases. These $B-V$ colors have also been found to be on average 0.02 mag bluer than the $B-V$ colors used for our data points and derived from Table 1; this may explain a small horizontal offset between the two datasets in Fig. 2. There was no attempt to merge the data into a larger and unique dataset, given the differences described above and the $U V$-selected origin of our data.

\section{Analysis}

\subsection{Context}

It is currently thought that HB stars and their progeny are responsible for the far- $U V$ emission in elliptical galaxies. The variety of observed $U V$ spectral energy distributions is explained by changing proportions of stars along the zero-age
HB (ZAHB) and the following post-HB evolution tracks. Three main classes of post-HB evolution are distinguished, each evolving from a different range of effective temperature on the ZAHB: from the red end of the HB, the stars evolve as postAGB stars, at hotter temperatures they follow post-early AGB evolution and the bluest follow AGB-Manqué evolution (e.g. Brown et al. 1998). The ZAHB location (hence the specific evolutionary track) is driven mainly by the envelope mass: the lower this mass, the hotter is the ZAHB location. The envelope mass itself depends critically on the mass losses during the red giant phase. The real populations involve a mixture of these different categories of stars. On one hand, the PAGB-stars fail to produce enough $U V$ because they are $U V$-bright for a too short period of time; nonetheless they are bright enough to be observed. On the other hand, the bluest HB stars and their progeny, if alone, would overproduce $U V$. The galaxies with stronger $U V$-upturn and bluer $U V$ color have therefore a larger fraction of their populations evolving along these latter paths and a smaller fraction of stars evolving along the PAGB track. Brown et al. (1997) have attempted to place numbers on these fractions. It is generally admitted that the strongest $U V$-upturn does not require more than about $15 \%$ of the evolving population passing through the hot $\mathrm{HB}$ phase. At the opposite, PAGB stars alone might account for the weakest $U V$ upturn. Nevertheless, STIS observations (Brown et al. 2000) have shown that the hot HB is populated in the case of the weak $U V$-upturn galaxy M 32 .

The situation is further complicated by the fact that, even if star formation is ruled out as a general interpretation of the $U V$ emission, it can still be present in a number of instances. Features in the optical spectra of some early-type galaxies have been interpreted as evidence for a low-level of star formation activity (e.g. Caldwell et al. 1993); NGC 205 and NGC 5102 are well known examples of nearby early-type galaxies with direct evidence for massive star formation (Hodge 1973; Pritchet 1979).

\subsection{Comparison with a sample of known objects}

A number of authors have developed spectral population synthesis models, based on the different families of evolutionary tracks for low-mass stars discussed above (e.g. Dorman et al. 1995; Tantalo et al. 1996; Yi et al. 1997). The $U V$ output is found to be extremely sensitive to the parameters used, chief among them the mass loss on the giant branch. Conversely, infering something on the various categories of low-mass stars responsible for the $U V$ flux is difficult, if not impossible, from observations as limited as a broad band measurement. Until the physical processes driving the distribution of stars along the ZAHB and the post-HB evolution are better understood our interpretation of $U V$ color will remain limited to analogies that can be established with a few objects used as references.

The BBBFL sample contains a significant number of known objects for which a correspondance can be made between the $U V-V$ color and the more detailed information available. They are NGC 1399 observed with HUT by Ferguson et al. (1991), the 6 early-type galaxies observed with HUT by 
Brown et al. (1995, 1997), M 31 and M 32 observed with HUT (Ferguson \& Davidsen 1993) and the HST (Brown et al. 1998, 2000 and references therein). NGC 205 and NGC 5102 have also been studied in details in the $U V$ domain (Bertola et al. 1995; Jones et al. 1996; Cappellari et al. 1999; Deharveng et al. 1997). All these 11 galaxies are identified in the color-color diagram of Fig. 2.

\subsection{The relative contribution of the different families of low-mass stars in advanced stages of evolution}

For the moment, we concentrate on the spread in $U V-V$ color, irrespectively of the scatter in $B-V$, and defer discussion of the bluest objects $(U V-V<1.5)$ close to NGC 205 $(U V-V=1.19)$ and NGC $5102(U V-V=0.8)$ to the following subsection. Most of the objects in Fig. 2 lie in the range of $U V$ colors $2-4.5$ defined by the two extreme objects, M $32(U V-V=4.5)$ and NGC $1399(U V-V=2.05)$ in the small sample of reference ellipticals. If the interpretation of NGC 1399 in terms of $U V$-bright stellar content is correct and can be extrapolated to other objects with the same $U V-V$ color, this means that in our relatively large sample we have not found galaxies requiring a larger population of hot HB than in NGC 1399, i.e. not more than say $15 \%$ of the evolving population passing through the hot HB. The precision in this number is of course limited by its extreme sensitivity to the envelope mass and the interplay of other parameters ( $Y, Z$, ages). At the opposite, the objects as red as 4.5 in $U V-V$ would be explained, if the case of M 32 is representative, essentially by PAGB evolution (although population passing through the hot HB phase has been resolved in M 32 by Brown et al. 2000). Of the 7 reddest objects, with $U V-V$ colors from 4.5 up to 5.5, 3 are among those in common with the BBBFL sample and reported with a disturbingly redder color than IUE data. These objects are likely to be affected by an integration of the $U V$ flux in a smaller area than in the optical band and remind us of the uncertainties (including systematics) in the color-color diagram. Although uncertain, these extreme red $U V$ colors would remain compatible with the interpretation by PAGB evolution, given the spread in envelope mass and the role of other parameters ( $Y, Z$ and ages) as illustrated by the models of Tantalo et al. (1996), Yi et al. (1997, 1998) and references therein.

An important feature of Fig. 2 is the large fraction of galaxies with $U V-V$ color in the bin $4 \pm 0.4$ : they make 29 of the 63 galaxies displayed in Fig. 2. They outnumber the objects in the bin $2.4 \pm 0.4$ by a factor 4 , or 36 when the difference of volume surveyed ( $U V$-flux limited sample) is roughly accounted for. They are not found from the same cluster as the unusually red early-type galaxies reported by Marcum et al. (2001) in the Perseus cluster. If our comparisons above are correct, this dominant number of galaxies with weak $U V$-upturn would imply that the PAGB stars which are necessarily present in elliptical galaxies are the main channel of evolution for the low-mass, metal-rich population. This large fraction of red objects, implying relatively young ages (e.g. Tantalo et al. 1996; Yi et al. 1998 ), is also consistent with the age spreads that are now being reported for early-type galaxies (e.g. Trager et al. 2000).
At the opposite, the scarcity of objects with $U V-V$ in the range 2.5-3.2 suggests a sort of bimodality along the ZAHB or something special with the group of six objects (including NGC 1399) reported with a strong $U V$-upturn with IUE.

Further interpretation, either on the possibility of a minor contribution of stars passing through the hot HB in the reddest galaxies, or on the factors controling the distribution at hot temperatures along the ZAHB, would require a larger number of objects and a better photometry than that offered by the present data.

\subsection{The case for recent star formation}

Our sample contains seven objects with $U V$ color bluer than 1.4, comparable to those of NGC $205(U V-V=1.19)$ and NGC $5102(U V-V=0.80)$. By analogy it is tempting to say these seven objects also harbour some residual star formation. While this conclusion seems reasonable for the bluest of these objects, it is more arbitrary for the others. There is indeed no solid argument to draw a line between galaxies with and without residual star formation. It is very possible that an $U V$ color slightly bluer than 2.0 may be explained by a more extreme than usual mixture of evolved low-mass stars and does not require any additional younger population. Conversely, it is conceivable that a low level of star formation can hide in a few objects with relatively red $U V$ colors.

We have examined individually the 7 objects with $U V-V<$ 1.4 and suspected star formation. Hints of star formation are known in three of them, CGCG 119053, CGCG 97125 and the bluest VCC 1499 (see notes to Table 1). VCC 49 (NGC 4168) has a low-luminosity Seyfert nucleus and CGCG 119030 reminds us that misclassification is also a possibility (see notes to Table 1). The two last, VCC 616 and CGCG 119086, have so far nothing special. This analysis reasonably confirms our assertion, based on the comparison with NGC 205 and NGC 5102, that the objects blue in $U V-V$ (say, <1.4) may have some residual star formation. It also suggests that the $U V$ light has the potential to sort out new cases of residual star formation.

If we now pay more attention to the location of the objects in the color-color diagram of Fig. 2, three (VCC 616, VCC 49 and CGCG 97125) of the 7 objects with $U V-V<1.4$ are puzzling by their relatively red $B-V$ whereas residual star formation is expected to move the objects to the blue in both colors. We have no explanations for this situation. The location of NGC 205 also deserves attention: its $B-V$ refers to the integrated light whereas the $U V-V$ from BBBFL refers to the central region; a $B-V$ of the order of 0.65 , as expected from the surface photometry of Lee (1996), would be more appropriate for the comparison. This would isolate further the three red $(B-V)$ objects discussed above.

For the 19 galaxies in Table 1 without an $U V-V$ color, the $U V-m_{\mathrm{pg}}$ color can be a useful approximation, especially for sorting out blue objects as discussed in this subsection. Of the five objects with $U V-m_{\mathrm{pg}}<0$ (VCC 1809, VCC 1111, CGCG 98078, VCC 914, VCC 288), one again (CGCG 98078) is known to have an extremely strong $\mathrm{H} \alpha$ emission (see notes to Table 1). 


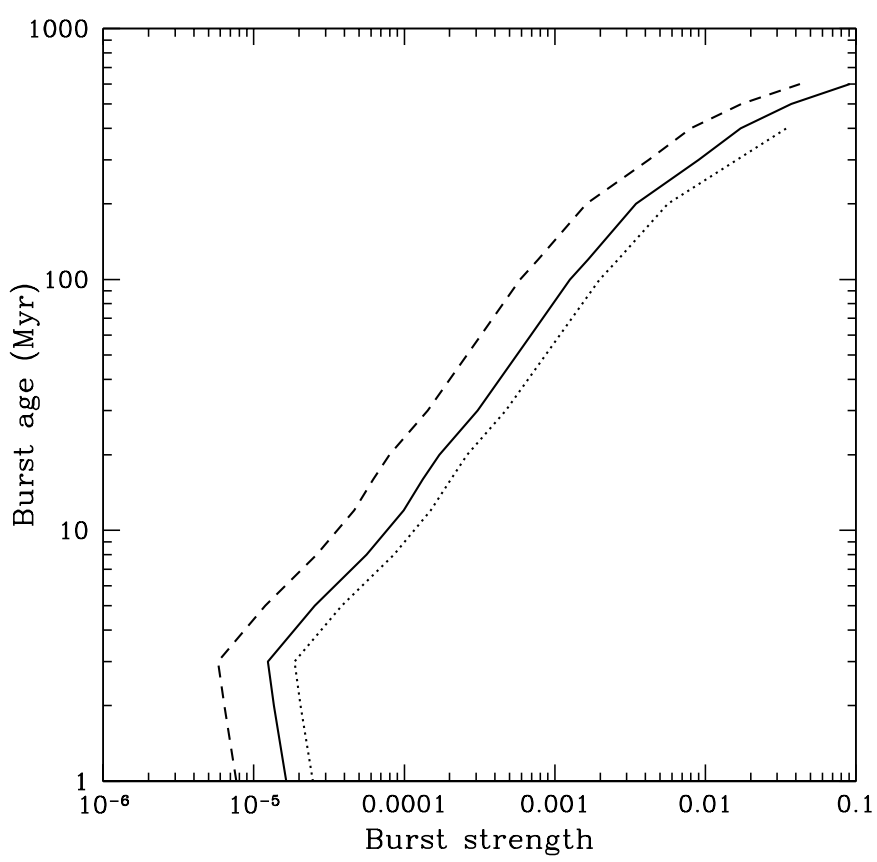

Fig. 3. The instantaneous bursts able to make an elliptical bluer than a given $U V-V$ color have their strengths and ages in the domain below and to the right of the curves. Solid line: composite color $<1.4$, host color $=4$; dashed line: same but host color $=2$; dotted line: composite color $<1$, host color $=4$. Solar metallicity and a Salpeter IMF (1-100 $M_{\odot}$ ) have been used in STARBURST 99 calculations.

It is difficult to translate such signs of on-going star formation into something more quantitative. For one, it is unknown whether the residual star formation takes place in an elliptical with a weak or a strong $U V$-upturn as caused by lowmass evolved stars. Second, the same $U V$ color excess may result from different combinations of burst strength, duration and ages. The situation is illustrated for an instantaneous burst in Fig. 3 displaying the combinations of strength and age that would make the $U V-V$ color of an host elliptical bluer than a given limit ( $U V-V$ of 1.4 and 1 adopted in Fig. 3). The time evolution of the spectral energy ditribution from the burst has been calculated with STARBURST 99 (Leitherer et al. 1999). A mass-to-visual light ratio of $7 M_{\odot} L_{V, \odot}^{-1}$ is assumed for the host elliptical (Charlot et al. 1996; Bressan et al. 1994), allowing us to define the burst strength as a fraction of the mass of the host and to calculate the composite color.

If a constant star formation is assumed, it is also possible with STARBURST 99 (Leitherer et al. 1999) to calculate the rate which would make an host elliptical bluer than a given $U V-V$ limit; the result has, however, to be expressed per unit $V$-band luminosity and becomes independent of duration for period over 100 Myrs. With the limit of $U V-V=1.4$ as in Fig. 3 we obtain $9.2 \times 10^{-12} M_{\odot} \mathrm{yr}^{-1} L_{V, \odot}^{-1}$ and $4.3 \times$ $10^{-12} M_{\odot} \mathrm{yr}^{-1} L_{V, \odot}^{-1}$ for an host with a $U V-V$ color of 4 and 2 respectively. For an elliptical with $M_{V}=-21$, this translates into rates of 0.2 and $0.09 M_{\odot} \mathrm{yr}^{-1}$ respectively. Such numbers are comparable with the rate of gas shed by stellar evolution in early-type galaxies (e.g. Faber \& Gallagher 1976). As noted by O'Connell (1999), a complete recycling into new stars is therefore excluded by $U V$ observations as a regular phenomenon in early-type galaxies. In contrast, the few objects bluer than the limits adopted suggest special events triggered by interaction and gas transfer. Constraints on a partial gas recycling would require to appreciate lower level of star formation, which is not yet permitted by the scatter of $U V$ colors and the present understanding of the $U V$ emission from $\mathrm{HB}$ stars and post-HB progeny.

\subsection{The scatter in $B-V$ and relationship to other properties}

An important feature of Fig. 2 is the significant number of objects that have relatively blue $B-V$ color (say $<0.85$ ), in contrast to the galaxies in the red vertical plume, as exemplified by the 4 reference objects (NGC 1399, NGC 4649, NGC 4552 and NGC 4486) which have red $B-V \sim 0.95$ (with $U V-V \sim 2.2$ ).

We have first examined whether a blueing in $B-V$ may be explained by the variety of evolution paths responsible for the scatter of the $U V$ color. We have not found any evidence for a blueing and a scatter of the $B-V$ color in the current stellar population models reproducing the $U V$ color (Bressan et al. 1994; Tantalo et al. 1996).

Residual star formation is a possible explanation, especially since the correlation between the $U V-V$ and $B-V$ extends into the domain of the bluest $U V-V$ galaxies that have been discussed in terms of star formation in the previous subsection. However, because the galaxies blue in $B-V$ are not all very blue in $U V-V$, it is also reasonable to consider other factors such as the diversity of luminosity and morphological type.

An individual examination of extreme objects is instructive. Of the seven objects with $B-V<0.75$ (and $U V-$ $V>1.5$ ), three are dwarf ellipticals (VCC 608, VCC 781, VCC 951), a category of objects known to have bluer $B-V$ than regular ellipticals (e.g. Ferguson 1994). Three are S0 galaxies (CGCG 127032, CGCG 160068, CGCG 160120); among them, CGCG 127032 (NGC 3821) is perhaps misclassified (see note to Table 1) and has a significant amount of neutral hydrogen (Eder et al. 1991), CGCG 160068 (NGC 4853) has strong Balmer absorption lines (see note to Table 1) and is the bluest in $U V-V$ of all seven. The last object (of the seven), VCC 1327 (NGC 4486A), should be discarded because a bright star is superimposed and probably contaminates the photometric measurements.

The potential role of the luminosity (and mass) has also been explored in various color-magnitude diagrams built with our sample. Figure 4 is an example of these diagrams with the $H$-band luminosity. The main trend of variation shows the blueing (and scatter) of the $B-V$ color with the low-luminosity objects, among them the three Virgo dwarf ellipticals discussed above. The branch leaving this main trend at $\log \left(L_{H}\right) \sim 11$ and $B-V \sim 0.75$ is caused by objects of the Coma cluster with possible misclassification due to the distance and signs of star formation as discussed above. Interestingly, this branch is comparable to that followed by spiral galaxies in a more general study of the photometric and structural properties of galaxies (Scodeggio et al. 2002). 


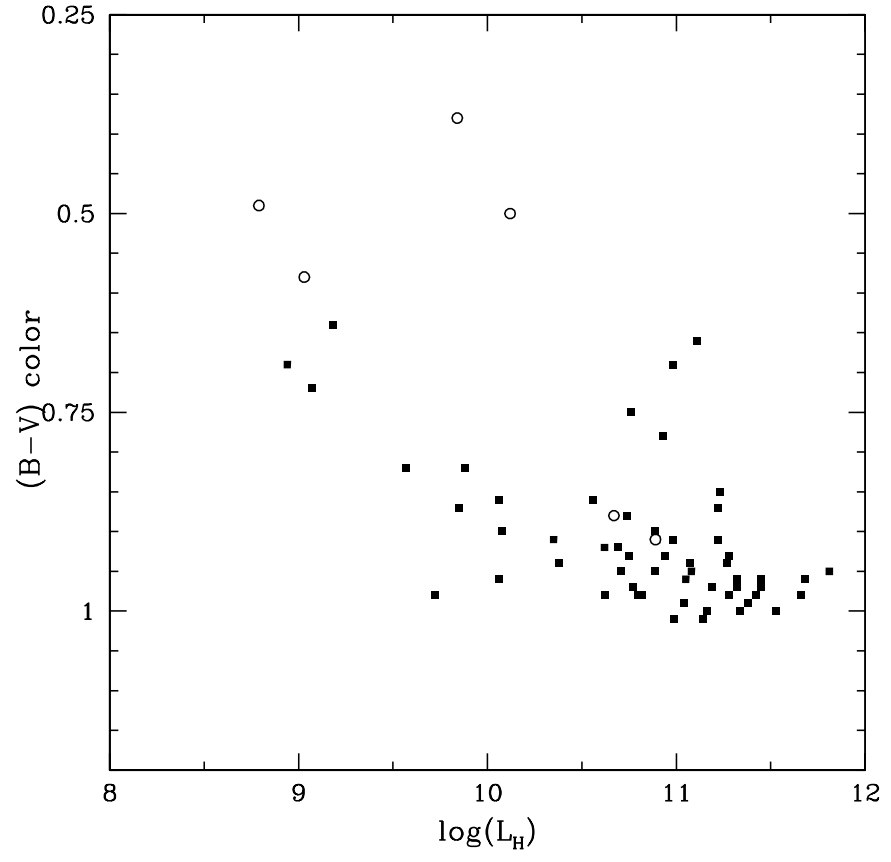

Fig. 4. $B-V$ vs. $H$ color-magnitude diagram for the galaxies of our sample (VCC 1327 has been discarded). The objects with $U V-V<$ 1.4 are plotted as open circles.

With an interpretation of the scatter in $B-V$ more in terms of luminosity effects than star formation (both are not exclusive), it remains to understand the apparent lack of galaxies blue in $B-V(<0.75$ as above) and red in $U V-V$ (say, $\sim 4)$. This feature contributes to enhance the correlation between the $U V-V$ and $B-V$ colors reported for a number of objects in the color-color diagram. There is probably a selection effect in the sense that a galaxy with $U V-V=4$ and a $B-V$ color implying an absolute magnitude $>-17$ would fall below the $U V$ detection limit at the distance of Virgo.

\subsection{The $\mathrm{Mg}_{2}-U V$ color correlation}

The correlation found between the $U V$ color and the spectral line index $\mathrm{Mg}_{2}$, with the color being bluer in more metal-rich galaxies (Faber 1983, BBBFL), has played a crucial role in establishing evolved low-mass stars rather than massive stars as the main source of $U V$ emission in early-type systems. In contrast, the 42 objects of our sample that have both an $U V-V$ color and a $\mathrm{Mg}_{2}$ index do not show such a correlation (Fig. 5). There are several possible explanations for this difference.

First, the $\mathrm{Mg}_{2}$ index obtained from the literature refers to the central regions whereas our $U V-V$ color refers to the integrated light. This may account for some of the scatter in Fig. 5 but not for the lack of correlation, as shown by the amplitudes of aperture correction displayed by Golev \& Prugniel (1998).

Second, the $\mathrm{Mg}_{2}-U V$ correlation of BBBFL is more apparent (as in Dorman et al. 1995) after removing galaxies with activity or on-going star formation. In such domains of the plot, we also have data points that might be removed on the same criteria.

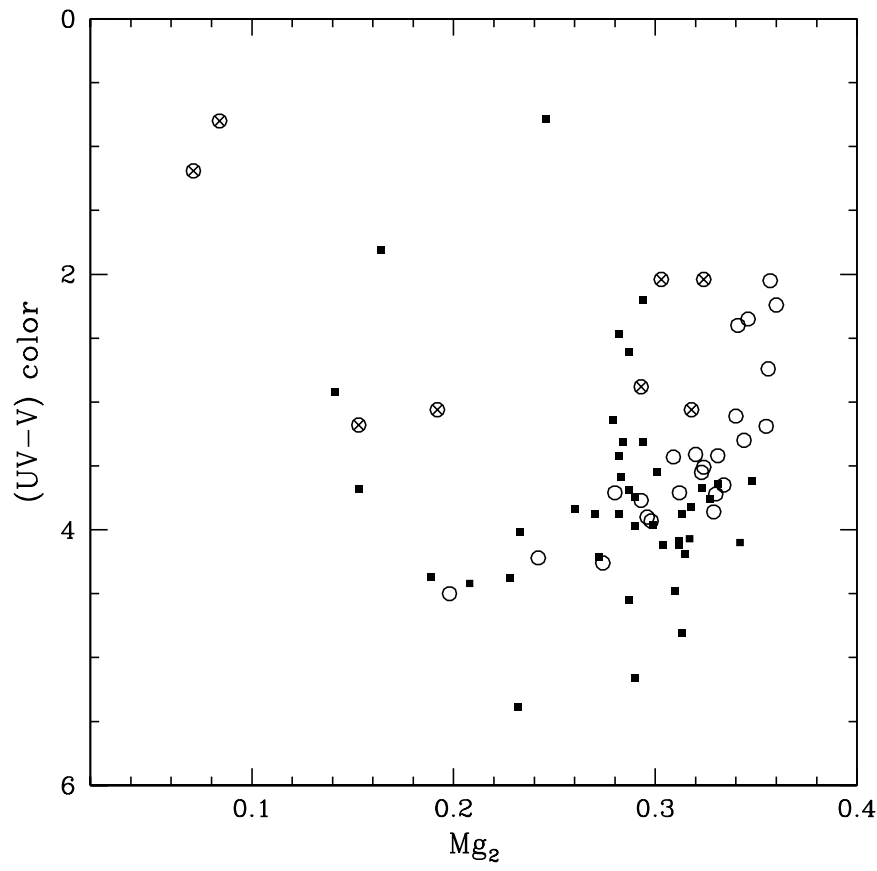

Fig. 5. The $U V-V$ color as a function of the $\mathrm{Mg}_{2}$ spectral line index. The sample of BBBFL is displayed for comparison (circles); their correlation is enhanced when galaxies with activity or on-going star formation (circles with diagonal crosses) are removed. The objects redder than 4.5 are among those discussed in Sects. 3 and 5.3 and may have their colors affected by aperture mismatch.

Third and more important, Fig. 5 shows that our data do not contradict the BBBFL correlation in terms of location in the plot but are clumped within a narrower domain than the BBBFL data. This feature, combined with the relatively large dispersion at a given $\mathrm{Mg}_{2}$ index, is in agreement with the idea (Dorman et al. 1995) that the correlation may arise from distinct classes of galaxies rather than from a continuum of properties. Most of our data would belong to an intermediate group with modest dependence of $U V$ colors on $\mathrm{Mg}_{2}$.

At the interpretation level the lack of correlation is not so embarrassing since the dominant factor for the production of $U V$ light is not the metallicity but the distribution of envelope masses on the ZAHB, which is itself determined by mass loss on the giant branch. The explanation for the correlation would be that mass-loss parameter increases with $Z$ (Dorman et al. 1995; O'Connell 1999). In contrast, the current correlation of the $B-V$ color with metal abundance, driven by opacity effects in stellar atmospheres, is equally clear in the two samples with the color getting redder as the $\mathrm{Mg}_{2}$ index increases.

\subsection{UV light profile and color gradient}

Four galaxies in our sample have a significant angular extent that allowed us to derive a radial $U V$ profile and $U V-B$ color profile, according to procedures described by Gavazzi et al. (2000b). The angular resolution of $\sim 20^{\prime \prime}$ in the $U V$ images prevents any conclusion to be reached within a radius of $20^{\prime \prime}$, a domain where most of the galaxies are known to exhibit a plateau in $U V-B$ color (Ohl et al. 1998). Beyond that radius 
and up to $50^{\prime \prime}$, the $U V-B$ color of M 87 displays a reddening of 0.7 mag comparable to that reported by Ohl et al. (1998) and then reaches a plateau up to $150^{\prime \prime}$, beyond the measurements with UIT (Ohl et al. 1998). In all three other objects (NGC 4374, NGC 4406 and NGC 4429) the angular extent is less and the external data points have low precision; NGC 4374 and NGC 4406 at least do not seem to support a reddening as reported by Ohl et al. (1998) in all their objects except M 32.

\section{Prospects for the GALEX survey}

The future GALEX survey (e.g. Martin et al. 1999) will considerably increase the number of flux measurements of galaxies in the far- $U V$. Adopting the counts of early-type systems of Marzke et al. (1998), assuming a limiting $U V$ magnitude of 18 and a simplified mixture of ellipticals with two $U V-V$ colors ( 4 and 2, in proportion 4 to 1 as in the present paper), we estimate that GALEX may detect about 4400 early-type galaxies in 20000 square degrees.

The sheer size of the sample would allow us to explore the role of many parameters in the $U V$ properties themselves. In combination with the uniformity of the $U V$-flux selection, it should be possible to expand over the two major issues addressed here, the relative proportion of the different categories of evolved stars responsible for the $U V$ emission and the relative frequency of residual star formation activity. From then and the measurement of the volume surveyed, it should be possible to estimate the volume density of star formation in early-type galaxies. Such an evaluation was not possible with our present sample because of the small number of objects, which in addition were mostly cluster galaxies.

The GALEX survey will also provide spectral information not available in the present sample, in the form of two $U V$ bands (and low-resolution spectra for some of the objects). Models based on two $U V$ bands and developed by Dorman et al. (1995), Yi et al. $(1997,1998)$ will help to take advantage of this additional information.

\section{Conclusions}

We have assembled a sample of 82 early-type galaxies with a flux measurement in the far-ultraviolet. In addition to more than doubling the number of objects, this sample has the advantage to be essentially $U V$-flux selected. The following has emerged from the analysis.

1) The large scatter of the $U V-V$ color in comparison with the colors in the optical is confirmed. As shown with a small number of objects studied previously in much detail, the color spread between 2 and 5 might be explained by changing proportions of stars along the ZAHB and the following postHB evolutionary tracks.

2) The galaxies with red $U V-V(\sim 4)$ colors (or weak $U V$ upturn) outnumber those with blue $U V-V(\sim 2)$ colors (or strong $U V$ upturn) in our sample. If the current interpretation of the $U V$-upturn can be extended to our sample, the PAGB tracks would be the most common evolution path among elliptical galaxies. Only a minority of elliptical galaxies would need a fraction of their stars evolving from the blue part of the ZAHB. The GALEX survey should considerably refine this finding, including possible differences between the various categories of early-type galaxies.

3) Few blue objects $(U V-V<1.5)$ may harbour some residual star formation as shown by the examples of NGC 205 and NGC 5102. The implication in terms of the cosmic density of the star formation rate in early-type galaxies should await for a more extended survey like GALEX.

4) For a fraction of the objects, the scatter of the $U V-V$ color is accompanied by a scatter in the $B-V$ color. The latter should be caused by the variety of morphological types and luminosities in the sample rather than the evolutionary features explaining the $U V$ emission.

5) The correlation between the $U V-V$ color and the $\mathrm{Mg}_{2}$ spectral index is not found. This is in line with the idea that the $U V$ flux is not driven by the metallicity but by the mass loss along the giant branch that determines the envelope mass on the horizontal branch.

Acknowledgements. We thank B. Milliard, M. Laget and M. Viton for providing new sets of $U V$ fluxes of galaxies in advance of publication, G. Gavazzi for sharing with us a large number of complementary photometric data, and A. Donati for running for us his program on the radial light profiles. The referee, R. O'Connell, is thanked for improvements to the text and for pointing out interesting features in the data.

\section{References}

Bertola, F., Bressan, A., Burstein, D., et al. 1995, ApJ, 438, 680

Binggeli, B., Sandage, A., \& Tammann, G. 1985, AJ, 90, 1681 (VCC)

Boselli, A., Tuffs, R., Gavazzi, G., Hippelein, H., \& Pierini, D. 1997, A\&A, 121, 507

Boselli, A., \& Gavazzi, G. 2002, A\&A, 386, 124

Bressan, A., Chiosi, C., \& Fagotto, F. 1994, ApJS, 94, 63

Brosch, N., Formiggini, L., Almoznino, E., et al. 1997, ApJS, 111, 143

Brosch, N. 1999, Experimental Astron., 9, 119

Brown, T. M., Ferguson, H. C., \& Davidsen, A. F. 1995, ApJ, 454, L15

Brown, T. M., Ferguson, H. C., Davidsen, A. F., \& Dorman, B. 1997, ApJ, 482, 685

Brown, T. M., Ferguson, H. C., Stanford, S. A., \& Deharveng, J.-M. 1998, ApJ, 504, 113

Brown, T. M., Bowers, C. W., Kimble, R. A., Sweigart, A. V., \& Ferguson, H. C. 2000, ApJ, 532, 308

Burstein, D., \& Heiles, C. 1982, AJ, 87, 1165

Burstein, D., Bertola, F., Buson, L. M., Faber, S. M., \& Lauer, T. R. 1988, ApJ, 328, 440 (BBBFL)

Caldwell, N., Rose, J., Sharples, R. M., Ellis, R. S., \& Bower, R. G. 1993, AJ, 106, 473

Caldwell, N., \& Rose, J. 1997, AJ, 113, 492

Cappellari, M., Bertola, F., Burstein, D., et al. 1999, ApJ, 515, L17

Charlot, S., Worthey, G., \& Bressan, A. 1996, ApJ, 457, 625

Code, A. D., Welch, G. A., \& Page, T. 1972, in The Scientific Results from the Orbiting Astronomical Observatory (OAO-2), ed. A. D. Code (NASA SP-310), 559

Code, A. D., \& Welch, G. A. 1981, ApJ, 228, 95

Code, A. D., \& Welch, G. A. 1982, ApJ, 256, 1

Corbin, M. R., Baldwin, J. A., \& Wilson, A. S. 1988, ApJ, 334, 584

Deharveng, J.-M., Sasseen, T., Buat, V., et al. 1994, A\&A, 289, 175 
Deharveng, J.-M, Jedrzejewski, R., Crane, P., Disney, M., \& Rocca-Volmerange, B. 1997, A\&A, 326, 528

Donas, J., Deharveng, J.-M., Milliard, B., Laget, M., \& Huguenin, D. 1987, A\&A, 180, 12

Donas, J., Buat, V., Milliard, B., \& Laget, M. 1990, A\&A, 235, 60

Donas, J., Milliard, B., \& Laget, M. 1995, A\&A, 303, 661

Dorman, B., O’Connell, R. W., \& Rood, R. T. 1995, ApJ, 442, 105

Eder, J., Giovanelli, R., \& Haynes, M. P. 1991, AJ, 102, 572

Faber, S. M. 1983, in Highlights of Astronomy, 6, 165

Faber, S. M., \& Gallagher, J. S. 1976, ApJ, 204, 365

Ferguson, H. C., Davidsen, A. F., Kriss, G. A., et al. 1991, ApJ, 382, L69

Ferguson, H. C., \& Davidsen, A. F. 1993, ApJ, 408, 92

Ferguson, H. C. 1994 In Dwarf Galaxies, ESO/OHP Workshop, ed. G. Meylan, \& P. Prugniel, 475

Gavazzi, G., \& Boselli, A. 1996, Astro. Lett. Commun., 35, 1

Gavazzi, G., Catinella, B., Carrasco, L., Boselli, A., \& Contursi, A. 1998, AJ, 115, 1745

Gavazzi, G., Boselli, A., Scodeggio, M., Pierini, D., \& Belsole, E. 1999a, MNRAS, 304, 595

Gavazzi, G., Carrasco, L., \& Galli, R. 1999b, A\&AS, 136, 227

Gavazzi, G., Franzetti, P., Scodeggio, M., Boselli, A., \& Pierini, D. 2000a, A\&AS, 142, 65

Gavazzi, G., Franzetti, P., Scodeggio, M., Boselli, A., \& Pierini, D. 2000b, A\&A, 361, 863

Gavazzi, G., Zibetti, S., Boselli, A., et al. 2001, A\&A, 372, 29

Golev, V., \& Prugniel, P. 1998, A\&AS, 132, 255

Greggio, L., \& Renzini, A. 1990, ApJ, 364, 35

Greggio, L., \& Renzini, A. 1999, Mem. Soc. Astron. It., 70, 691

Ho, L. C., Filippenko, A. V., Sargent, W. L. W., \& Peng, C. Y. 1997, ApJS, 112, 391

Hodge, P. W. 1973, ApJ, 182, 671

Jones, D. H., Mould, J. R., Watson, A. M., et al. 1996, ApJ, 466, 742

Jorgensen, I. 1999, MNRAS, 306, 607

Kennicutt, R., \& Kent, S. 1983, AJ, 88, 1094
Kennicutt, R., Bothun, G. D., \& Schommer, R. A. 1984, AJ, 89, 1279 Lee, M. G. 1996, AJ, 112, 1438

Leitherer, C., Schaerer, D., Goldader, J. D., et al. 1999, ApJS, 123, 3

Maoz, D., Filippenko, A. V., Ho, L. C., et al. 1996, ApJS, 107, 215

Marcum, P. M., O’Connell, R. W., Fanelli, M. N., et al. 2001, ApJS, 132,129

Martin, C. D., Bianchi, L., Donas, J., et al. 1999, In Ultraviolet-Optical Space Astronomy Beyond HST, ed. J. A. Morse, J. M. Shull, \& A. L. Kinney, ASP Conf., 164, 182

Marzke, R. O., Da Costa, L. N., Pellegrini, P. S., et al. 1998, ApJ, 503, 617

Milliard, B., Donas, J., \& Laget, M. 1991, Adv. Space Res., 11, 135

Moss, C., Whittle, M., \& Pesce, J. 1998, MNRAS, 300, 205

O'Connell, R. W., Bohlin, R. C., Collins, N. R., et al. 1992, ApJ, 395, L45

O'Connell, R. W. 1999, ARA\&A, 37, 603

Ohl, R. G., O’Connell, R. W., Bohlin, R. C., et al. 1998, ApJ, 505, L11

Pritchet, C. 1979, ApJ, 231, 354

Prugniel, P., Nieto, J.-L., \& Simien, F. 1987, A\&A, 173, 49

Rifatto, A., Longo, G., \& Capaccioli, M. 1995, A\&AS, 114, 527

Scodeggio, M., Gavazzi, G., Franzetti, P., et al. 2002, A\&A, 384, 812

Smith, A. M., \& Cornett, R. H. 1982, ApJ, 261, 1

Sparke, L. S., Kormendy, J., \& Spinrad, H. 1980, ApJ, 235, 755

Tantalo, R., Chiosi, C., Bressan, A., \& Fagotto, F. 1996, A\&A, 311, 361

Trager, S. C., Faber, S. M., Wortey, G., \& González, J. J. 2000, AJ, 119,1645

Trinchieri, G., \& Di Serego Alighieri, S. 1991, AJ, 101, 1647

Worthey, G. 1994, ApJS, 95, 107

Yi, S., Demarque, P., \& Oemler, A. 1997, ApJ, 486, 201

Yi, S., Demarque, P., \& Oemler, A. 1998, ApJ, 492, 480

Zwicky, F., Herzog, E., Karpowicz, M., Kowal, C., \& Wild, P. 19611968, Catalogue of Galaxies and of Cluster of Galaxies (Pasadena, California Institute of Technology; CGCG) 